\title{
A new interacting multiple model particle filter based ballistic missile tracking method
}

\author{
Liyun Gong \\ School of Computer Science \\ University of Lincoln, UK \\ Email: lgong@lincoln.ac.uk
}

\author{
Miao Yu \\ School of Computer Science \\ University of Lincoln, UK \\ Email: myu@lincoln.ac.uk
}

\begin{abstract}
This paper proposes a new method for tracking the whole trajectory of a ballistic missile from launch to impact on the ground. Multiple state models are applied for the ballistic missile movement descriptions during different phases, while the transition probabilities are modelled in a state-dependent way. A radar sensor is applied to obtain the missile range, azimuth angle and elevation angle measurements. Based on the state models and measurements, an interacting multiple model based particle filter method is applied for tracking. Simulation studies show that the proposed method outperforms the widely-applied extended Kalman filtering based interacting multiple model for tracking the ballistic missile.
\end{abstract}

Index Terms-ballistic missile tracking, multiple model, particle filtering, state dependent

\section{INTRODUCTION}

The ballistic missile (BM) is one of the threats from the air in modern warfare, and it is important to intercept it against the related attack. To intercept the BM, firstly it needs to be tracked to obtain its position and velocity. Various works have been done with respect to the BM tracking. The tracking of re-entry $\mathrm{BM}$ is proposed in [1$3]$. Based on the re-entry state model and measurements, Extended Interval Kalman filter [1] and sequential Monte Carlo based approach [2] are applied for tracking the BM. A comparison study between different filtering methods for spiralling missile tracking during re-entry phase is proposed in [3]. From the simulation results, it is shown that the Rao-Blackwellized particle filter achieves the best performance especially when large initial uncertainties are introduced. Y. Li et al. [4] studies the problem of estimating the BM trajectory during the boost phase. Based on the line-of-sight measurements, an optimization based method is applied to obtain the BM state at a reference time from which the trajectory could be further predicted.

In reality, a BM experiences different phases from its launch to impact and the movement characteristics in different phases are different. To accurately track the whole trajectory of the BM considering different movement characteristics, multiple state models are needed.
Currently, the most widely used method for the multiple model based BM tracking is the interacting multiple model (IMM) method as in [5] and [6]. At each time instance, the filtering results from multiple models are combined to give the final estimation. However, there is an unrealistic constant transition probabilities assumption for the traditional IMM. Besides, the extended Kalman filter applied in the IMM scheme has a limitation in dealing with highly non-linear models related to BM tracking.

In order to overcome these limitations, an interactive multiple model particle filtering (IMMPF) algorithm as in [7] is applied for BM tracking. This algorithm is based on exact Bayesian inference for the state estimation. Compared with the IMM, more realistic state dependent model transition probabilities are considered and a particle filtering approach is applied to overcome the nonlinearities of both the state and measurement models. The structure of this paper is as follows: Section 2 gives detailed descriptions of the state models and measurement model used in BM tracking. An illustration of the IMMPF algorithm is presented in Section 3. Simulation studies are presented in Section 4. Finally, we give the conclusions in Section 5.

\section{BM TRACKING MODELS}

In this section, we introduce both the state and measurement models for tracking the whole trajectory of a BM. These models are used for the algorithm development in Section III.

\section{A. State models}

The entire trajectory of a BM target from launch to impact is commonly divided into three phases [8]-[9]: boost phase, coast phase and re-entry phase. Three state models are then applied to reflect different BM dynamics in different phases. We consider a more realistic scenario with unknown BM parameters exist and then model related them together with state for each state model. 
1) Boost phase model: According to the Newton's force law, the following basic equations hold:

$$
\dot{\mathbf{p}}=\mathbf{v} \quad \dot{\mathbf{v}}=\mathbf{a}
$$

where $\mathbf{p}=\left[p_{x}, p_{y}, p_{z}\right], \mathbf{v}=\left[v_{x}, v_{y}, v_{z}\right]$ and $\mathbf{a}=$ $\left[a_{x}, a_{y}, a_{z}\right]$ represent the position, velocity and acceleration vectors representatively in the earth-centered-earthfixed (ECEF) coordinate system [8]. For simplicity, the subscript representing time is omitted.

During the boost phase, the BM's ignition is on and it is in the lower part of the atmosphere. The BM is affected by: the gravity force $\mathbf{f}_{\text {gravity }}$, thrust force $\mathbf{f}_{\text {thrust }}$, aerodynamic drag force $\mathbf{f}_{d r a g}$ as well as additional Coriolis force $\mathbf{f}_{\text {coriolis }}$ and Centripetal force $\mathbf{f}_{\text {centripetal }}$ introduced by the earth's rotation. According to the force definitions in [9], the acceleration components along the three axes of the ECEF coordinate system are represented in (2). As defined in [9], $u_{G}, g$ and $\omega$ are constant values. $\rho(\cdot)$ is defined as an exponential function with respect to the $\mathrm{BM}$ altitude $h . n, q$ and $\beta$ represent the initial thrust-to-weight ratio, normalized mass burn rate and ballistic coefficient respectively, which are usually unknown.

$$
\begin{aligned}
a_{x} & =\frac{n g}{1-q t} \frac{v_{x}}{\sqrt{v_{x}^{2}+v_{y}^{2}+v_{z}^{2}}}-\frac{\rho(h)}{2 \beta} v_{x} \sqrt{v_{x}^{2}+v_{y}^{2}+v_{z}^{2}} \\
& -\frac{u_{G}}{\left(\sqrt{p_{x}^{2}+p_{y}^{2}+p_{z}^{2}}\right)^{3}} p_{x}+\left(2 \omega v_{y}+\omega^{2} p_{x}\right) \\
a_{y} & =\frac{n g}{1-q t} \frac{v_{y}}{\sqrt{v_{x}^{2}+v_{y}^{2}+v_{z}^{2}}}-\frac{\rho(h)}{2 \beta} v_{y} \sqrt{v_{x}^{2}+v_{y}^{2}+v_{z}^{2}} \\
& -\frac{u_{G}}{\left(\sqrt{p_{x}^{2}+p_{y}^{2}+p_{z}^{2}}\right)^{3}} p_{y}+\left(2 \omega v_{x}+\omega^{2} p_{y}\right) \\
a_{z} & =\frac{n g}{1-q t} \frac{v_{z}}{\sqrt{v_{x}^{2}+v_{y}^{2}+v_{z}^{2}}}-\frac{\rho(h)}{2 \beta} v_{z} \sqrt{v_{x}^{2}+v_{y}^{2}+v_{z}^{2}} \\
& -\frac{u_{G}}{\left(\sqrt{p_{x}^{2}+p_{y}^{2}+p_{z}^{2}}\right)^{3}} p_{z} .
\end{aligned}
$$

According to (1)-(2) and by applying the Wiener process model [8] for modeling unknown ballistic parameters, we can obtain the following nineth-order state model as:

$$
\left[\begin{array}{c}
\dot{p}_{x} \\
\dot{p}_{y} \\
\dot{p}_{z} \\
\dot{v}_{x} \\
\dot{v}_{y} \\
\dot{v}_{z} \\
\dot{\beta} \\
\dot{n} \\
\dot{q}
\end{array}\right]=\left[\begin{array}{c}
v_{x} \\
v_{y} \\
v_{z} \\
a_{x} \\
a_{y} \\
a_{z} \\
0 \\
0 \\
0
\end{array}\right]+\mathbf{w}_{\text {boost }}
$$

where $\mathbf{w}_{\text {boost }}$ is a $9 \times 1$ vector representing the uncertainty.

2) Coast model: During the coast phase, the BM usually follows a Keplerian orbit at higher parts of or even outside the atmosphere, with the ignitions being off. In this case, it will not be affected by the thrust force and drag forces and the corresponding acceleration components then become:

$$
\begin{aligned}
& a_{x}=-\frac{u_{G}}{\left(\sqrt{p_{x}^{2}+p_{y}^{2}+p_{z}^{2}}\right)^{3}} p_{x}+\left(2 \omega v_{y}+\omega^{2} p_{x}\right) \\
& a_{y}=-\frac{u_{G}}{\left(\sqrt{p_{x}^{2}+p_{y}^{2}+p_{z}^{2}}\right)^{3}} p_{y}+\left(2 \omega v_{x}+\omega^{2} p_{y}\right) \\
& a_{z}=-\frac{u_{G}}{\left(\sqrt{p_{x}^{2}+p_{y}^{2}+p_{z}^{2}}\right)^{3}} p_{z} .
\end{aligned}
$$

We can see that the acceleration terms do not contain any ballistic missile parameters due to the negligible thrust and drag forces. Without considering any BM parameters ( $n$, $q$ and $\beta$ ), the related state model reduces to a sixth-order one as:

$$
\left[\begin{array}{c}
\dot{p}_{x} \\
\dot{p}_{y} \\
\dot{p}_{z} \\
\dot{v}_{x} \\
\dot{v}_{y} \\
\dot{v}_{z}
\end{array}\right]=\left[\begin{array}{c}
v_{x} \\
v_{y} \\
v_{z} \\
a_{x} \\
a_{y} \\
a_{z}
\end{array}\right]+\mathbf{w}_{\text {coast }}
$$

where $\mathbf{w}_{\text {coast }}$ is a $6 \times 1$ vector representing the uncertainty.

3) Reentry model: When the BM re-enters the low parts of the atmosphere (troposphere and stratosphere), it suffers from the drag force again, and the related acceleration terms becomes:

$$
\begin{aligned}
a_{x} & =-\frac{\rho(h)}{2 \beta} v_{x} \sqrt{v_{x}^{2}+v_{y}^{2}+v_{z}^{2}}-\frac{u_{G}}{\left(\sqrt{p_{x}^{2}+p_{y}^{2}+p_{z}^{2}}\right)^{3}} p_{x} \\
& +\left(2 \omega v_{y}+\omega^{2} p_{x}\right) \\
a_{y} & =-\frac{\rho(h)}{2 \beta} v_{y} \sqrt{v_{x}^{2}+v_{y}^{2}+v_{z}^{2}}-\frac{u_{G}}{\left(\sqrt{p_{x}^{2}+p_{y}^{2}+p_{z}^{2}}\right)^{3}} p_{y} \\
& +\left(2 \omega v_{x}+\omega^{2} p_{y}\right) \\
a_{z} & =-\frac{\rho(h)}{2 \beta} v_{z} \sqrt{v_{x}^{2}+v_{y}^{2}+v_{z}^{2}}-\frac{u_{G}}{\left(\sqrt{p_{x}^{2}+p_{y}^{2}+p_{z}^{2}}\right)^{3}} p_{z} .
\end{aligned}
$$

A seventh-order state model is applied to model the position, velocity and ballistic coefficient for estimation as:

$$
\left[\begin{array}{c}
\dot{p}_{x} \\
\dot{p}_{y} \\
\dot{p}_{z} \\
\dot{v}_{x} \\
\dot{v}_{y} \\
\dot{v}_{z} \\
\dot{\beta}_{t}
\end{array}\right]=\left[\begin{array}{l}
v_{x} \\
v_{y} \\
v_{z} \\
a_{x} \\
a_{y} \\
a_{z} \\
0
\end{array}\right]+\mathbf{w}_{\text {reentry }}
$$

where $\mathbf{w}_{\text {reentry }}$ is a $7 \times 1$ vector representing the uncertainty.

\section{B. Measurement model}

The BM could be measured by one or more radar sensors. Normally, sensors measure the range $r$, azimuth angle $\theta$ and elevation angle $\varphi$. The measurement model 
equations are represented as:

$$
\begin{aligned}
& r=\sqrt{\left(p_{x}-p_{x}^{R}\right)^{2}+\left(p_{y}-p_{y}^{R}\right)^{2}+\left(p_{z}-p_{z}^{R}\right)^{2}}+n_{r} \\
& \theta=\arctan \left(\frac{p_{y}-p_{y}^{R}}{p_{x}-p_{x}^{R}}\right)+n_{\theta} \\
& \varphi=\arctan \left(\frac{p_{z}-p_{z}^{R}}{\sqrt{\left(p_{x}-p_{x}^{R}\right)^{2}+\left(p_{y}-p_{y}^{R}\right)^{2}}}\right)+n_{\varphi}
\end{aligned}
$$

where $\left[p_{x}^{R}, p_{y}^{R}, p_{z}^{R}\right]$ represents the radar position. $n_{r}, n_{\theta}$ and $n_{\varphi}$ represent the noises of the measurement model.

\section{ALGORITHM DESCRIPTION}

Based on different models, the IMMPF algorithm [7] is applied for the ballistic missile tracking. Compared with the traditional IMM method, it considers more realistic state-dependent transition probabilities and the particle filtering based implementation makes it robust to the high non-linearity of both the state and measurement models. The IMMPF algorithm is based on the exact Bayesian inference framework for a multiple model system, whose overall process is divided into four steps: mode mixing, state interaction, evolution and correction.

Mode mixing: The mode mixing is related to the evolution of the model probability between consecutive discrete time instances $t-1$ and $t$. Using the law of total probability, we have:

$$
\begin{aligned}
& p\left(m_{t}=s \mid \mathbf{Z}_{t-1}\right)=\sum_{r \in \mathcal{M}} p\left(m_{t}=s, m_{t-1}=r \mid \mathbf{Z}_{t-1}\right) \\
& =\sum_{r \in \mathcal{M}} p\left(m_{t}=s \mid m_{t-1}=r, \mathbf{Z}_{t-1}\right) p\left(m_{t-1}=r \mid \mathbf{Z}_{t-1}\right),
\end{aligned}
$$

where $m_{t}$ represents the state model index variable whose value could be any one element in the set $\mathcal{M}=$ \{boost, coast,

reentry\}. $\mathbf{Z}_{t-1}$ represents the measurements collection during previous time instances. And $p\left(m_{t}=s \mid m_{t-1}=\right.$ $\left.r, \mathbf{Z}_{t-1}\right)$ can further be decomposed as:

$$
\begin{aligned}
& p\left(m_{t}=s \mid m_{t-1}=r, \mathbf{Z}_{t-1}\right) \\
& =\int \pi_{r s}\left(\mathbf{x}_{t-1}\right) \cdot p\left(\mathbf{x}_{t-1} \mid m_{t-1}=r, \mathbf{Z}_{t-1}\right) \mathrm{d} \mathbf{x}_{t-1} .
\end{aligned}
$$

where $\mathbf{x}_{t-1}$ represents the BM state vector containing position, velocity and possible missile parameters. $\pi_{r s}\left(\mathbf{x}_{t-1}\right)$ represents the state-dependent model transition probability between models $r$ and $s$. For the BM tracking in this work, we apply Wu's polynomial function [10] to model transition probabilities with respect to the BM height information.

State intersection: State interaction generates the initial mode-conditioned density $p\left(\mathbf{x}_{t-1} \mid m_{t}=s, \mathbf{Z}_{t-1}\right)$. According to the conditional probability relation and the law of total probability, one has:

$p\left(\mathbf{x}_{t-1} \mid m_{t}=s, \mathbf{Z}_{t-1}\right)=\frac{\sum_{r \in \mathcal{M}} \pi_{r s}\left(\mathbf{x}_{t-1}\right) \cdot p\left(\mathbf{x}_{t-1}, m_{t-1}=r \mid \mathbf{Z}_{t-1}\right)}{p\left(m_{t}=s \mid \mathbf{Z}_{t-1}\right)}$.

Evolution: The state evolution step is to propagate the mode-conditioned state density from $t-1$ to $t$. Given the initial density is provided in (11), the mode-conditioned prior distribution $p\left(\mathbf{x}_{t} \mid m_{t}=s, \mathbf{Z}_{t-1}\right)$ at $t$ can be calculated as:

$$
\begin{aligned}
& p\left(\mathbf{x}_{t} \mid m_{t}=s, \mathbf{Z}_{t-1}\right) \\
& =\int p\left(\mathbf{x}_{t} \mid \mathbf{x}_{t-1}, m_{t}=s, \mathbf{Z}_{t-1}\right) p\left(\mathbf{x}_{t-1} \mid m_{t}=s, \mathbf{Z}_{t-1}\right) \mathrm{d} \mathbf{x}_{t-1} .
\end{aligned}
$$

Correction: Finally, the updated measurement is incorporated to correct the prior by following the Bayes' rule:

$$
\begin{aligned}
p\left(\mathbf{x}_{t}, m_{t}=s \mid \mathbf{Z}_{t}\right) & \propto p\left(\mathbf{z}_{t} \mid \mathbf{x}_{t}, m_{t}=s\right) p\left(\mathbf{x}_{t} \mid m_{t}=s, \mathbf{Z}_{t-1}\right) \\
& \cdot p\left(m_{t}=s \mid \mathbf{Z}_{t-1}\right) .
\end{aligned}
$$

\section{A. IMMPF implementation}

Since there is no analytical solution for the proposed Bayesian filter due to the nonlinearity and non-Gaussian distribution of the multiple model system, a particle filter is developed to implement such a filtering framework. It starts at time $t-1$ with the set of weighted particles $\left\{\mathbf{x}_{t-1}^{r, k}, \mu_{t-1}^{r, k} ; r \in \mathcal{M}, k \in\{1, \ldots, N\}\right\}$ to approximate the probability $p\left(\mathbf{x}_{t-1}, m_{t-1}=r \mid \mathbf{Z}_{t-1}\right)$, based on which prior mode probability in (9) is approximated as

$$
p\left(m_{t}=s \mid \mathbf{Z}_{t-1}\right) \approx \sum_{m_{k-1} \in \mathcal{M}} \sum_{k=1}^{N} \pi_{r s}\left(\mathbf{x}_{k-1}^{r, k}\right) \cdot \mu_{t-1}^{r, k} \triangleq \Lambda_{t-1}^{s},
$$

where $\Lambda_{t-1}^{s}$ is defined to facilitate the rest of derivation.

Secondly, the state interaction process can be implemented by inserting particles into (11), such that

$$
\begin{aligned}
& p\left(\mathbf{x}_{t-1} \mid m_{t}=s, \mathbf{Z}_{t-1}\right) \\
& \approx \sum_{r \in \mathcal{M}} \sum_{k=1}^{N} \pi_{r s}\left(\mathbf{x}_{t-1}^{r, k}\right) \mu_{t-1}^{r, k} \delta\left(\mathbf{x}_{t-1}-\mathbf{x}_{t-1}^{r, k}\right) / \Lambda_{t-1}^{s} .
\end{aligned}
$$

The density $p\left(\mathbf{x}_{t-1} \mid m_{t}, \mathbf{Z}_{t-1}\right)$ is the initial density for the mode-matched filter. From (15), we can see it is approximated by a number of $|\mathcal{M}| \times N$ (where $|\mathcal{M}|$ is the number of models) particles, which will lead to the exponentially increasing of particles for initial density representation. To solve this problem, resampling is performed such that $N$ samples $\left\{\mathbf{x}_{t-1}^{s, k}\right\} \sim p\left(\mathbf{x}_{t-1} \mid m_{t}=s, \mathbf{Z}_{t-1}\right)$ are generated.

The third step is the particles' evolution from time $t-1$ to $t$. Resampled particle set $\left\{\mathbf{x}_{t-1}^{s, k}\right\}_{k=1, \ldots, N}$ could be evolved to generate a new particle set $\left\{\mathbf{x}_{t}^{s, k},\right\}_{k=1, \ldots, N} \sim$ 
$p\left(\mathbf{x}_{t} \mid m_{t}=s, \mathbf{Z}_{t-1}\right)$, with the aid of the state model corresponding to $m_{t}=s$. We have to notice that the state models in (3), (5) and (7) are represented in continuous forms. In order to evolve particles at discrete time instances from $t-1$ to $t$, we apply the Euler method [11] (more complicated Runge-Kutta method [11] could also be adopted). The generated new particle set could be applied to approximate the predicted distribution $p\left(\mathbf{x}_{t} \mid m_{t}=s, \mathbf{Z}_{t-1}\right)$ as:

$$
p\left(\mathbf{x}_{t} \mid m_{t}, \mathbf{Z}_{t-1}\right) \approx \frac{1}{N} \sum_{k=1}^{N} \delta\left(\mathbf{x}_{t}-\mathbf{x}_{t}^{s, k}\right) .
$$

Finally, by inserting (14) and (16) into (13), we have:

$$
\begin{aligned}
p\left(\mathbf{x}_{t}, m_{t} \mid \mathbf{Z}_{t}\right) & \propto \sum_{k=1}^{N} \frac{\Lambda_{t-1}^{s}}{N} p\left(\mathbf{z}_{t} \mid \mathbf{x}_{t}^{s, k}, m_{t}=s\right) \delta\left(\mathbf{x}_{t}-\mathbf{x}_{t}^{s, k}\right) \\
& \propto \sum_{k=1}^{N} \mu_{t}^{s, k} \delta\left(\mathbf{x}_{t}-\mathbf{x}_{t}^{s, k}\right) .
\end{aligned}
$$

We can see that the distribution $p\left(\mathbf{x}_{t}, m_{t}=s \mid \mathbf{Z}_{t}\right)$ could be approximated by a set of new particles $\mathbf{x}_{t}^{s, k}$ with weights $\mu_{t}^{s, k} \propto p\left(\mathbf{z}_{t} \mid \mathbf{x}_{t}^{s, k}, m_{t}=s\right) \Lambda_{t-1}^{s}$, from which the state model probability for $p\left(m_{t}=s \mid \mathbf{Z}_{t}\right)$ and final state estimation $\hat{\mathbf{x}}_{t}$ is obtained as as:

$$
\begin{gathered}
\hat{\mathbf{x}}_{t}=\sum_{s \in \mathcal{M}} \sum_{k=1}^{N} \mu_{t}^{s, k} \mathbf{x}_{t}^{s, k}, \\
p\left(m_{t}=s \mid \mathbf{Z}_{t}\right)=\sum_{k=1}^{N} \mu_{t}^{s, k} .
\end{gathered}
$$

\section{Simulation STUdies}

To verify the effectiveness of the IMMPF algorithm and its advantages over the traditional IMM algorithm ( [5] and [7]) for the ballistic missile tracking, a simulation study is performed. A whole BM trajectory is simulated in the Earth-centred-Earth-fixed (ECEF) coordinate system as in Fig. 1 by three state models as mentioned in Section 2. It is simulated that the BM transits from boosting to coasting at $66 \mathrm{~s}$ after launching and from coasting to reentering into the lower atmosphere region at $275 \mathrm{~s}$. Key parameters of the simulated BM flight trajectory are listed in Table I, which fall into the short range ballistic missile trajectory parameters as described in [12]. A radar sensor positioned at $29^{\circ} \mathrm{N}$ and $42^{\circ} \mathrm{E}$ (corresponding to the position $\left[3.96 \times 10^{6}, 4.02 \times 10^{6}, 3.09 \times 10^{6}\right]$ (meters) in the ECEF coordinate system) is applied to measure the range, azimuth and elevation angles of the BM.

Based on the simulated scenario, 100 Monte-Carlo simulations are performed to compare the performance of IMM and IMMPF (with the particle size being 5000 for each state model). Firstly, we compare the BM state

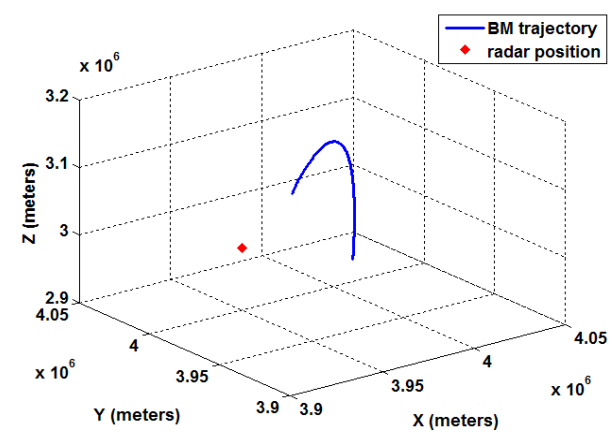

Fig. 1. Simulated BM trajectory

TABLE I

THE PARAMETERS OF THE SIMULATED BM TRAJECTORY

\begin{tabular}{|c|c|c|c|}
\hline Flight time & Range & Boost time & Engine-off velocity \\
\hline $305(\mathrm{~s})$ & $292(\mathrm{~km})$ & $66(\mathrm{~s})$ & $1.46(\mathrm{~km} / \mathrm{s})$ \\
\hline
\end{tabular}

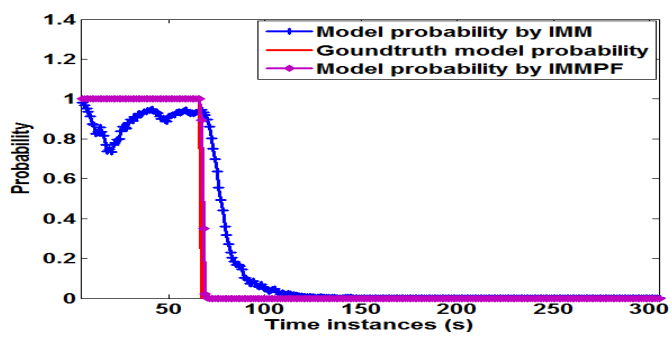

(a) Boost model probability estimation

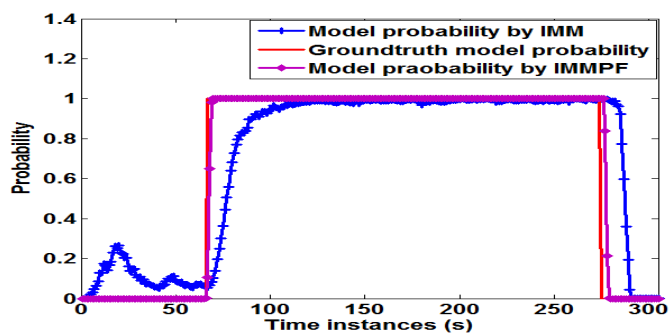

(b) Coast model probability estimation

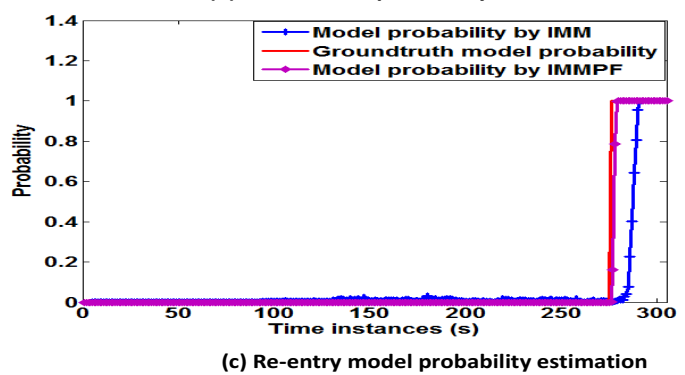

Fig. 2. The comparisons on the estimations of different state model probabilities. 
model probability estimations by different methods and the results are presented in Fig. 2. We can see that the probability estimations of IMMPF coincide with the ground truth much closer and the IMMPF algorithm could better capture the BM dynamic type changes with less time delay compared with the traditional IMM.

Next we compare the averaged root-mean-square-error (RMSE) of two methods, for both position and velocity. From Fig.3, we could observe that for the IMM algorithm, large errors could be observed around instances when model transitions occur, and this could be largely reduced by the IMMPF algorithm which better captures the BM dynamic model transitions as shown in Fig.2. Besides, by applying the particle filtering based approach, the nonlinearities of both state and measurement models are better handled thus more accurate estimations could be obtained within a particular phase.

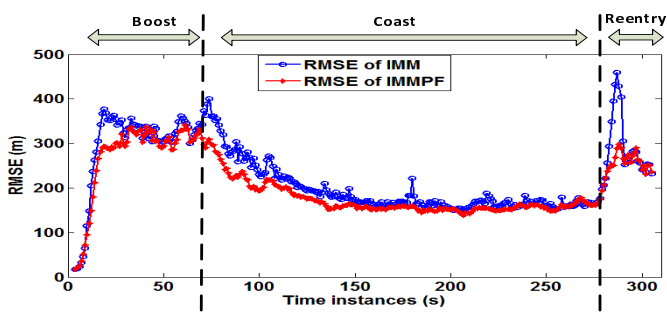

(a) Position RMSEs

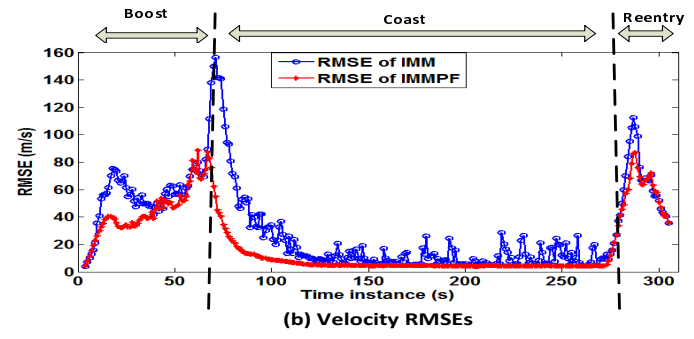

Fig. 3. The comparisons on RMSEs for positions and velocities

\section{Conclusions}

In this paper, we have proposed a new IMMPF based method for tracking the whole trajectory of the ballistic missile. Multiple models were applied for describing ballistic missile movements during different phases, with the state-dependent model transition probabilities being applied. This particle filtering based method can better deal with the non-linearities of both the state and measurement models. The simulations showed that the IMMPF outperforms the traditional IMM for the estimations of both the model probability and state vector components.

\section{REFERENCES}

[1] G. Siouris, G. Chen, and J. Wang, "Tracking an Incoming Ballistic Missile Using an Extended Interval Kalman Filter," IEEE
Transactions on Aerospace and Electronic Systems, vol. 33, no. 1, pp. 232-240, 1997.

[2] M. Bruno and A. Pavlov, "Improved Sequential Monte Carlo Filtering for Ballistic Target Tracking," IEEE Transactions on Aerospace and Electronic Systems, vol. 41, no. 3, pp. 1103-1108, 2005.

[3] J. Kim, S. Vaddi, S. Menon, and E. Ohlmeyer, "Comparison Between Nonlinear Filtering Techniques for Spiraling Ballistic Missile State Estimation," IEEE Transactions on Aerospace and Electronic Systems, vol. 48, no. 3, pp. 313-328, 2012.

[4] Y. Li, T. Kirubarajan, Y. Bar-Shalom, and M. Yeddanapudi, "Trajectory and Launch Point Estimation for Ballistic Missiles from Boost Phase LOS Measurements," in IEEE Aerospace Conference, Snowmass at Aspen, CO, USA, 1999.

[5] R. Cooperman, "Tactical ballistic missile tracking using the interacting multiple model algorithm," in The Fifth International Conference on Information Fusion,Annapolis, MD, USA, 2002.

[6] W. Farrell, "Tracking of a Ballistic Missile with A-Priori Information," IEEE Transactions on Aerospace and Electronic Systems, vol. 44, no. 2, pp. 418-426, 2008.

[7] H. Blom and E. Bloem, "Exact Bayesian and Particle Filtering of Stochastic Hybrid Systems," IEEE Transactions on Aerospace and Electronic Systems, vol. 43, no. 1, pp. 55-70, 2007.

[8] X. Li and V. Jilkov, "Survey of Maneuvering Target Tracking. Part ii: Motion Models of Ballistic and Space Targets," IEEE Transactions on Aerospace and Electronic Systems, vol. 46, no. 1, pp. $96-119,2010$

[9] A. Benavoli, L. Chisci, and A. Farina, "Tracking of a Ballistic Missile with A-Priori Information," IEEE Transactions on Aerospace and Electronic Systems, vol. 43, no. 3, pp. 1000-1016, 2007.

[10] Z. Wu, "Compactly Supported Positive Definite Radial Functions," Advances in Computational Mathematics, vol. 4, no. 1, pp. 283292, 1995.

[11] R. Burden and J. Faires, "Numerical analysis (5th ed.)," $P W S$ Publishing, Boston, USA, 1993.

[12] "https://www.gov.uk/government/publications/cde-themedcompetition-defence-against-airborne-threats," accessed in September, 2015. 